\title{
The measurement and interpretation of genotype by environment interaction in spring barley (Hordeum vulgare)
}

\author{
W. Powell,* P. D. S. Caligari,* \\ M. S. Phillips* and J. L. Jinks $\dagger$
}

* Scottish Crop Research Institute, Pentlandfield, Roslin, Midlothian, EH25 9RF, Scotland.

$\dagger$ Department of Genetics, University of Birmingham, Birmingham, B15 2TT.

The linear regressions of individual genotypes on the mean value of all genotypes for a number of environments has been used as a measure of a genotype's response to environmental variables. The present data from spring barley indicated that genotype by environment interactions may not always be adequately explained by a linear function of the environment. A genotype's phenotypic variance over environments may be used as a measure of environmental sensitivity. It is demonstrated that randomly produced $F_{3}$ and or doubled haploid families may be used to predict new combinations of mean performance and environmental sensitivity.

\section{INTRODUCTION}

Plant breeders are only too aware of the problems caused by genotype by environment interactions $(G \times E)$. Such interactions are revealed in a statistical sense by a significant interaction term when two or more genotypes are grown in two or more contrasting environments. This statistical definition, however, covers a wide range of diverse biological phenomena. In order to have a better understanding of genotype by environment interaction, joint regression analysis (Yates and Cochran, 1938) has been applied to trial data. This technique, which was developed and advanced by Finlay and Wilkinson (1963) as well as Jinks and co-workers (Perkins and Jinks, $1968 a, b$; Jinks and Pooni, 1980), involves quantifying each environment by the means of all the genotypes in the experiment. For each genotype the linear regression of individual values on the environmental means is then calculated. The salient feature of this analysis is that the environments are measured in biological terms and not specified by physical factors. The sums of squares measuring the interaction item may then be partitioned into an heterogeneity term which measures the differences between the slopes of the regressions and a deviation term which measures the scatter of points about the regression lines. Although the joint regression approach has been used extensively (Breese, 1969; Lawes, 1977;
Kaltsikes and Larter, 1970) its value as a measure of adaptability is dependent on a high degree of linearity. There is evidence from a number of crops that mean performance and sensitivity to macroenvironmental variables are highly correlated. Examples are yield in maize (Eberhart and Russell, 1966) and final height in Nicotiana rustica (Jinks and Connolly, 1975). The present study was conducted on spring barley to assess the linear regression approach as a means of measuring a genotype's environmental sensitivity. The relationship between mean expression and environmental sensitivity in random inbred lines of spring barley was also investigated.

\section{MATERIALS AND METHODS}

Three spring barley pair crosses were studied:

Golden Promise $\times$ Mazurka (TT1)

Golden Promise $\times$ Ark Royal (TT3)

BH4/143/2 × Ark Royal (TT4)

The experiment in 1983 included: the parents, $F_{2}$ and $F_{3}$ generations for the TT1, TT3 and TT4 crosses. Also included were $20 \mathrm{~F}_{1}$ derived double haploid $(\mathrm{DH})$ lines and 40 single seed descent (SSD) lines. Sowing data and density were manipulated to produce four environments. The first environment $\left(S_{1} 10\right)$ was created by sowing ten seeds per family with a $5 \mathrm{~cm}$ spacing between plants. Rows were spaced $22.5 \mathrm{~cm}$ apart with a 
wheat "guard" sown at each end of each row. On the same day, on an adjacent plot of land, the $S_{1} 5$ experiment was sown in exactly the same manner as for the $S_{1} 10$ experiment but in this case there were only five plants per row which produced a spacing of $10 \mathrm{~cm}$ between plants. Fifteen days later the second series $\left(S_{2}\right)$ of experiments were sown to produce the $S_{2} 10$ and $S_{2} 5$ environments.

The experimental design was a randomised complete block of two replicates and the experiments were netted to prevent bird damage. The following characters were scored:

1. Awn emergence, days from the 1st of June until awns emerged from the flag leaf sheath. (AE).

2. Maturity (Mat) scored on a 1 to 9 scale (1:early, 9:late).

3. Final height, measured from the base of the plant to the collar in $\mathrm{cm}(\mathrm{Ht})$.

4. The number of fertile tillers per plant $(\mathrm{TN})$.

5. The number of grains per ear, measured on the main system $(\mathrm{GN})$.

6. The yield of grain on the main stem in $\mathrm{gm}^{-2}$ (MSW).

7. The length of the ear in $\mathrm{cm}$ (EL).

8. Thousand grain weight calculated using GN and MSW (TGW).

9. Grain yield of the whole plant, single plant yield (SPY).

\section{RESULTS}

The results of the joint regression analysis for the three crosses are given in table 1 . In most cases both the heterogeneity and deviations items are significant which indicate that some of the interaction with environment can be explained in terms of a linear response with the environment but that there are also deviations from linearity which cannot be explained by experimental error. A convenient way to examine the relative contribution of the heterogeneity and deviation items to $G \times E$ is to use the components of variance. These are given in table 2 and in some cases $e . g$, awn emergence in the TT1 cross all the $G \times E$ may be explained by the deviation component. In contrast main stem weight in the TT1 cross a linear relationship can adequately explain the $G \times E$ interaction. Finlay and Wilkinson (1963) who originally used this method to compare the yield performance of a set of cereal varieties grown at different sites found a high degree of linearity and used the estimates of the regression slopes to measure adaptability. The present data on barley clearly demonstrate that it is not reasonable to simply assume that the $G \times E$ interactions may be explained by a linear function of the environment.

$M S W$ in the TT1 cross which displays a linear and hence predictable response will be used to illustrate the phenotypic regression approach. It should be noted that since the regression coefficients are based on the means of all genotypes, the average response must have a regression coefficient of 1.0 (Mather and Jinks, 1982). The phenotypic expression $(Y)$ of a particular genotype $(i)$ in a specific environment $(j)$ depends on three genetic properties: a mean performance $(\mu)$, a linear response to the environment $\left[(1+\beta) a_{j}\right]$ and residual deviations from regression $\left(S_{i j}\right)$.

$$
Y_{i j}=\mu_{i}+\left(1+\beta_{i}\right) a_{j}+S_{i j}
$$

Table 1 Joint regression analyses of the TT1, TT3 and TT4 crosses

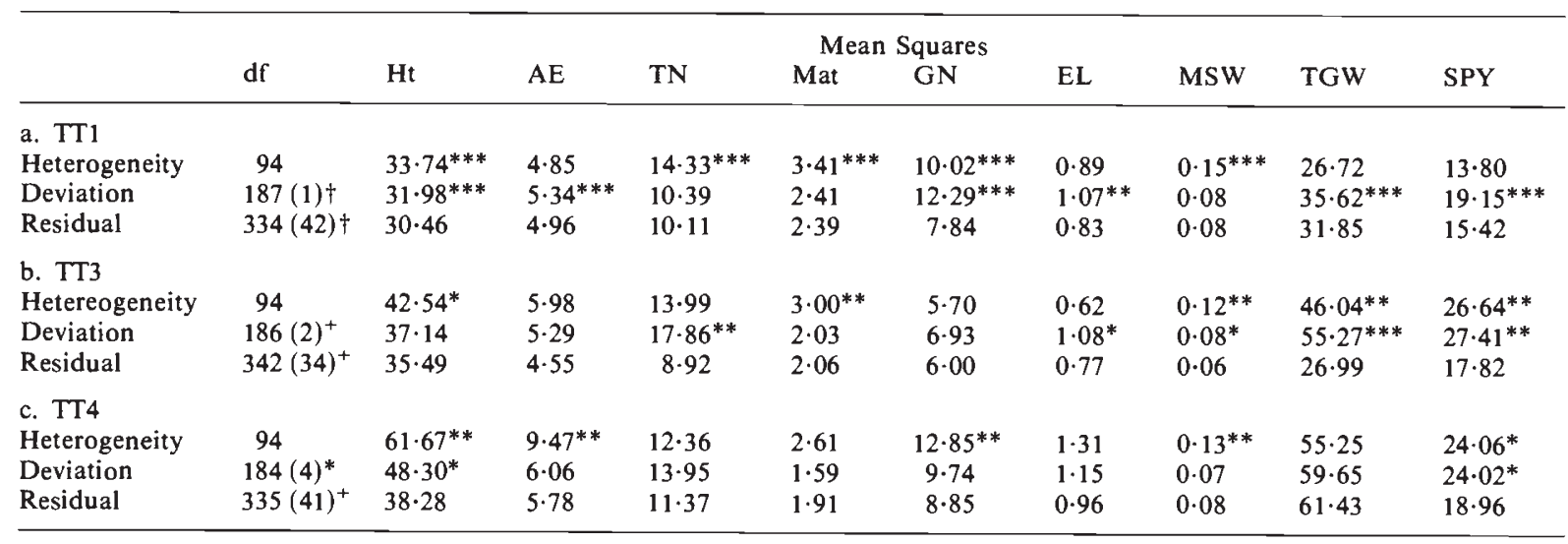

$\dagger$ indicates number of missing values 
Table 2 Components of variance attributable to heterogeneity and deviations expressed as percentages

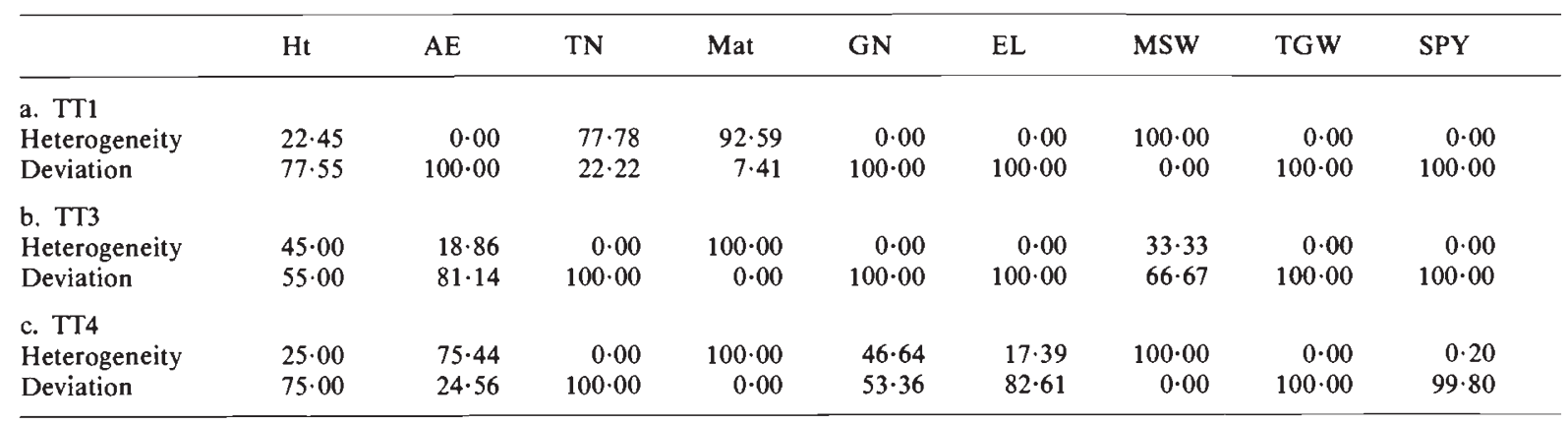

It is therefore clear that individual means, the regression slope and the deviations around the slope must all be considered in evaluating the potential performance of genotypes. The remainder M.S. for each individual genotype from the TT1 cross are very variable and reflect the fact that mean squares measuring the scatter of points about individual regression lines are not homogeneous $\left(\chi_{[86]}^{2}=120 \cdot 46, P<0 \cdot 001\right.$ using Bartlett's test $)$. The mean square measuring the overall deviations from regressions presented in table 1 are therefore not strictly valid. Furthermore the deviations from regression appear to be under genetic control and hence characteristic of particular genotypes, as was found by Mather (1975).

Various stability parameters have been suggested by numerous workers (for a review see Hill, 1975) but the most useful method has been proposed by Jinks (1976). The basic statistics needed for this approach are the mean performance of a genotype in respect of any trait averaged over all environments and variation in performance over these environments. These two measures will be used in subsequent analyses and provide a two dimensional assessment of each genotype for each character. Environmental sensitivity being measured as the square root of the variance $(\sigma)$ component over environments.

The phenotypic correlations between mean performance and $\sigma$ for the three crosses are given in table 3. Non-significant correlations, e.g. $M S W$, indicate independence of the genes controlling mean performance and environmental sensitivity. A significant correlation between mean performance and $\sigma$ on the other hand indicates pleiotropy and or linkage disequilibrium in the control of these characters. Since DH and SSD samples will differ in terms of the number of rounds of recombination a comparison of the correlation coefficients in these two populations may offer a means of distinguishing between these two relationships (Caligari, Powell and Jinks 1985a). In the case of $T N$ significant correlations exist in both the $\mathrm{DH}$ and SSD samples and indicate a pleiotropic

Table 3 Phenotypic correlations between mean performance and regression slope $(\beta)$, upper figure, as well as mean performance and square root of the variance $(\sigma)$, lower figure, for the TT1, TT3 and TT4 crosses

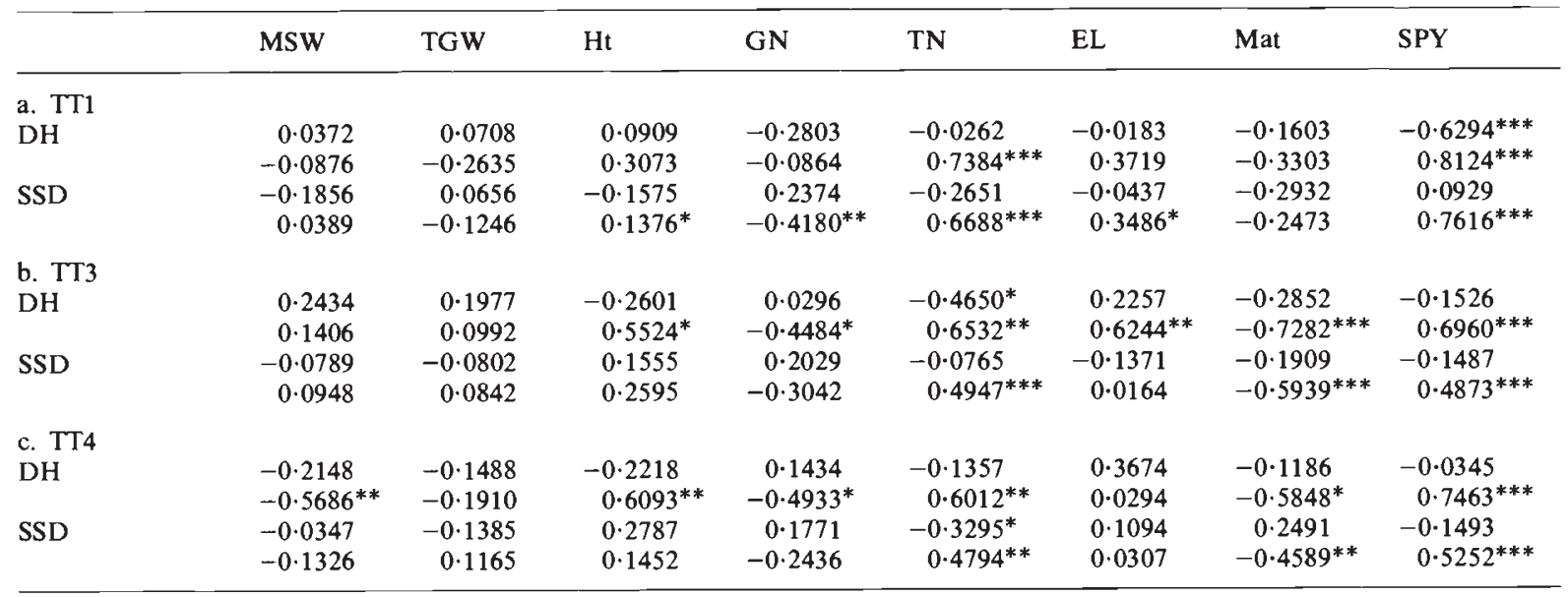


relationship between mean performance and environmental sensitivity or tight linkage. Similarly tight linkage or pleiotropy may also explain the positive correlation between yield and environmental sensitivity as measured as the square root of the variance component. In practice these two explanations are indistinguishable where independent genetical systems are physically tightly linked. However, the use of the regression slope $(\beta)$ to measure environmental sensitivity may be totally misleading and this may be illustrated by inspection of figs. 1 and 2. In fig. 1 the slopes are plotted against mean performance (for SPY) and the nonsignificant correlation is in striking contrast to that observed in fig. 2 where the square root of the vairance component replaces the slope. In the case of height in the TT4 cross a significant positive correlation exists in the DH sample but not in the SSD sample. Clearly there is an excess of coupling linkages in the DH generation which are broken down following rounds of recombinations. The association between tall genotypes and above average sensitivities may be broken following opportunities for recombination.
The three crosses used in the present study contain the erectoides dwarfing gene and it has been demonstrated that this locus affects the expression of quantitative characters (Powell et al., $1985 a$ ). Furthermore, the method used to establish an association between major genes and agronomic characters may be extended and applied to the phenotypic variance of any given character. The principle of the method depends on the ability to classify inbred lines into two groups: an erect group which possess the erectoides dwarfing allele and the tall (nutans) group which possesses the alternative allele. It is thus possible to assess the effects of the erectoides locus on the square root of the variance component. The appropriate analyses of variance for the DH and SSD populations are given in tables 4(a) and 4(b). The mean squares for the erectoides $\mathrm{v}$ nutans item in the $\mathrm{DH}$ population are only significant for awn emergence and height in the TT4 cross. Thus in these cases the means of the two sub-populations are significantly different. Furthermore the fact that these mean squares are significant when tested against the between lines within groups item indicates that

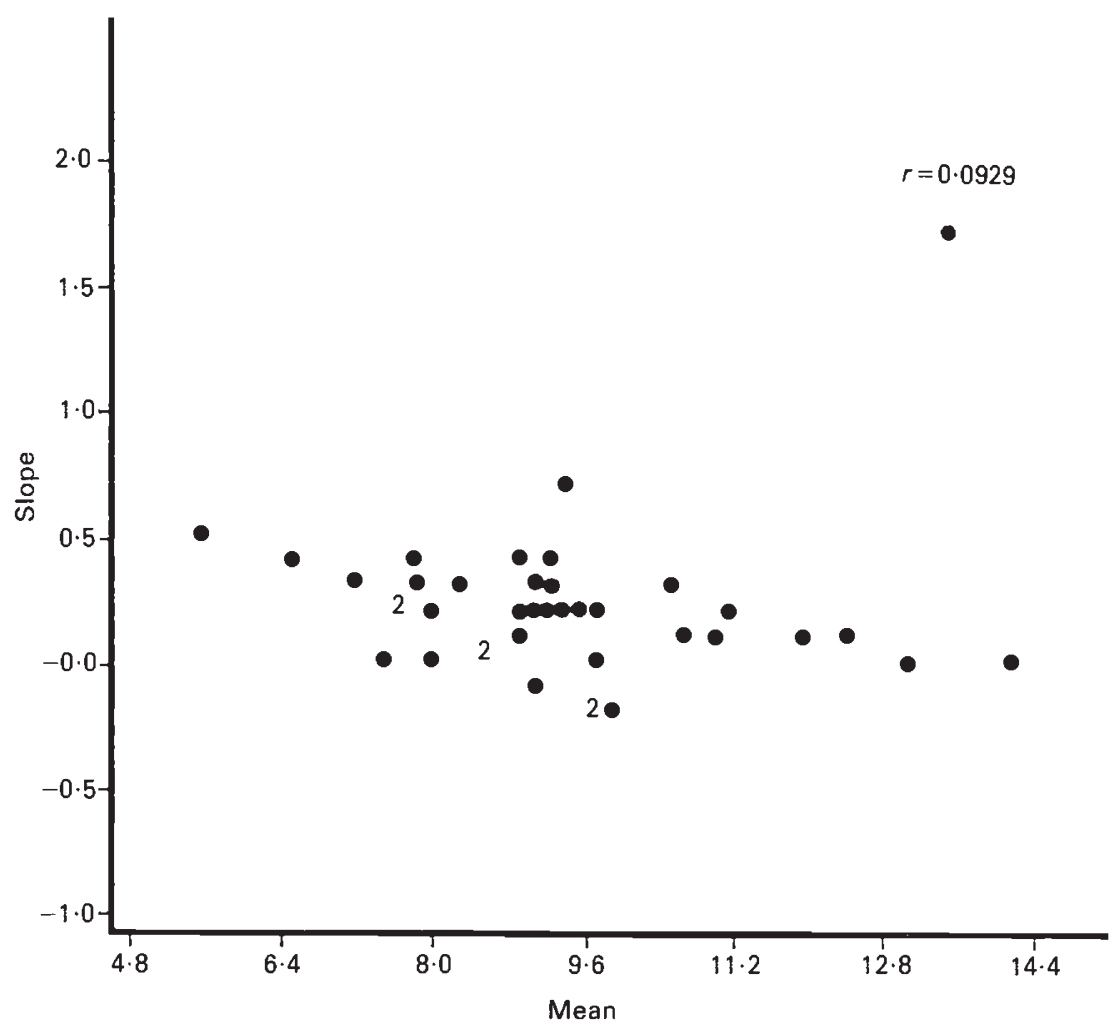

Figure 1 Relationship between mean and slope for SPY in the SSD sample for the TT1 cross. The 2's plotted on the graph indicate the coincidence of two data items. 


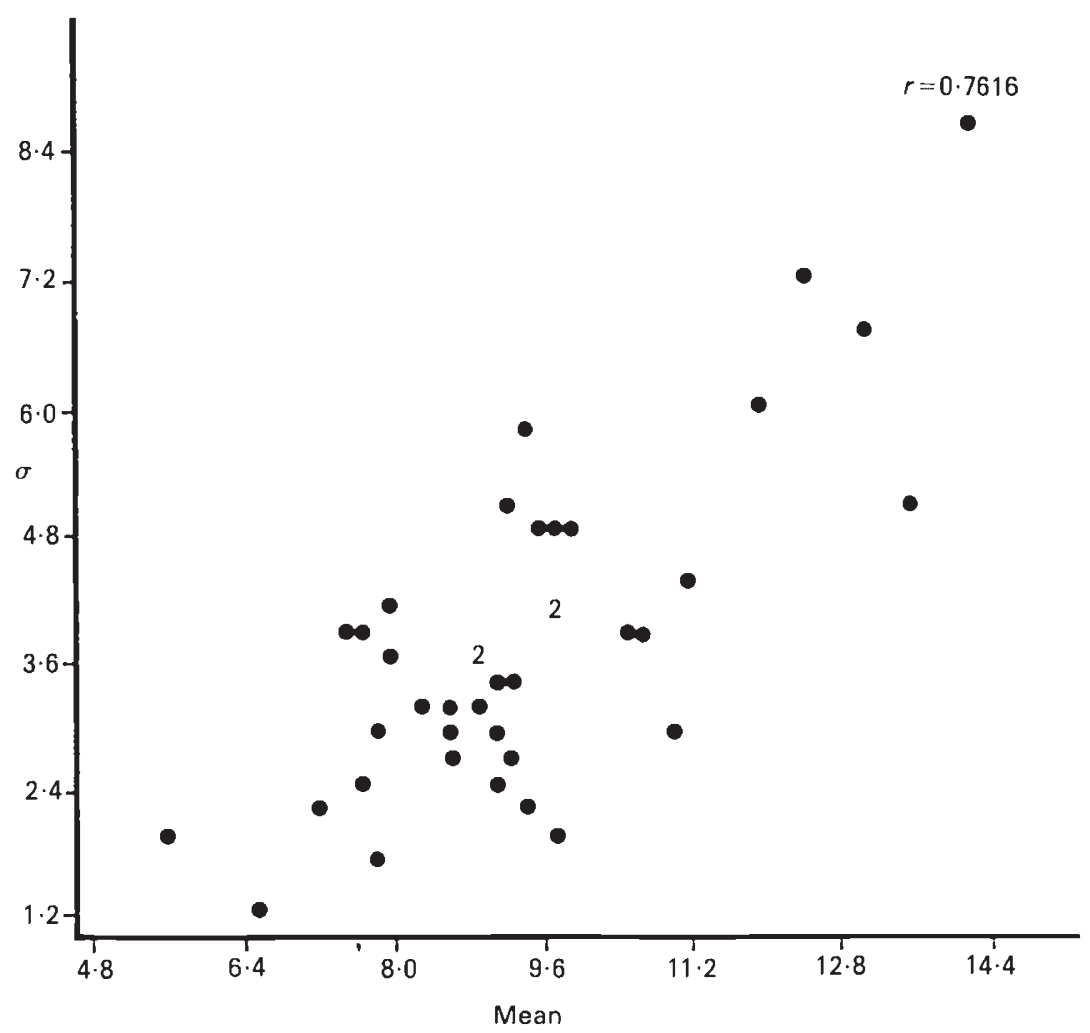

Figure 2 Relationship between mean and $\sigma$ for SPY in the SSD sample for the TT1 cross. The 2's plotted on the graph indicate the coincidence of two data items.

a significant portion of the additive genetic variation $(D)$ is associated with allelic differences at the GP ert locus. This association may be due to pleiotropy and or linkage disequilibrium. Inspection of table 4(b) indicates that for these characters in the TT4 cross there are no significant differences between the two sub-populations in the SSD generation. It is therefore likely that the association between mean performance and environmental sensitivity in these cases is due to linkage which is broken following rounds of recombination. There are significant differences between the erect and nutans sub-populations in the TT3 cross (SSD sample) for height and $M S W$ at the 5 per cent level. Although only border line in significance this suggests an association between the erectoides locus and environmental sensitivity. This may be due to pleiotropy or tight linkage which has not been broken following extra rounds of gametogenesis.

It has been shown that the properties of recombinant inbred lines may be predicted from the early generations of a cross (Caligari et al., 1985a; Powell et al., 1985b). By replicating genotypes over four environments it is possible to make predic- tions for sensitivity to macro-environmental differences (Jinks and Pooni, 1980). Estimates of $m$, the mean of all possible inbred lines and $\sqrt{D}$, the standard error of the means of all possible inbred families have been calculated from the DH and $F_{3}$ samples. This information is summarised in table 5 for environmental sensitivity (where there is significant genetic variation). The univariate predictions derived from this information by the methods outlined by Jinks and Pooni (1976) are given for the TT1 and TT3 crosses in table 5(b). The predicted numbers can be compared with observed numbers for environmental sensitivity. Both predictions and observations are given as whole numbers summing to 40 , the total number of SSD lines. It is clear from these tables that it is possible to predict the number of inbreds falling into defined phenotypic classes i.e., environmental sensitivity can be included as a character in cross prediction programmes and estimates of genetical parameters obtained from the early generations of a cross may be used to predict the frequency of transgressive segregants.

It is possible to predict the joint distribution of two characters simultaneously (Jinks and Pooni, 
Table 4 Results of the analyses of variance for environmental sensitivity

$\begin{array}{llllllllll} & \text { AE } & \text { EL } & \text { GN } & \text { Ht } & \text { Mat } & \text { MSW }\left(10^{2}\right) & \text { TGW } & \text { TN } & \text { SPY } \\ \text { df } & \text { MS } & \text { MS } & \text { MS } & \text { MS } & \text { MS } & \text { MS } & \text { MS } & \text { MS } & \text { MS }\end{array}$

(a) the DH samples

TT1

1. Between reps

2. Between groups (ert vs. nutans)

3. Bet. lines within groups

4. Reps $\times$ lines

TT3

1. Between reps

2. Between groups (ert vs. nutans)

3. Bet. lines within groups

4. Reps $\times$ lines

TT4

1. Between reps

2. Between groups (ert vs. nutans)

3. Bet. lines within groups

4. Reps $\times$ lines

18

\begin{tabular}{|c|c|c|c|c|c|c|c|c|c|}
\hline 1 & $2 \cdot 31$ & $0 \cdot 28$ & $2 \cdot 20$ & 0.61 & $1 \cdot 21$ & 0.68 & $23 \cdot 80$ & 0.07 & 1.88 \\
\hline 1 & $2 \cdot 15$ & $0 \cdot 68$ & $1 \cdot 05$ & $2 \cdot 20$ & 0.92 & 0.52 & 0.92 & 0.54 & $0 \cdot 28$ \\
\hline 18 & 1.42 & $0 \cdot 18$ & $2 \cdot 26$ & $5 \cdot 41$ & 0.53 & 1.05 & $9 \cdot 33$ & $2 \cdot 44$ & $4 \cdot 36$ \\
\hline 19 & $1 \cdot 61$ & $0 \cdot 15$ & $2 \cdot 39$ & $2 \cdot 00$ & $2 \cdot 00$ & $1 \cdot 21$ & $7 \cdot 43$ & $2 \cdot 28$ & $3 \cdot 17$ \\
\hline 1 & 1.61 & $0 \cdot 20$ & $4 \cdot 34$ & 0.09 & $0 \cdot 13$ & $5 \cdot 11$ & 58.08 & 0.22 & $4 \cdot 42$ \\
\hline 1 & 0.68 & $0 \cdot 15$ & $1 \cdot 74$ & $2 \cdot 49$ & $1 \cdot 00$ & $3 \cdot 01$ & $16 \cdot 25$ & $0 \cdot 29$ & 1.02 \\
\hline 18 & 0.60 & $2 \cdot 13$ & $2 \cdot 97$ & $11 \cdot 90$ & 0.79 & $3 \cdot 39$ & $18 \cdot 19$ & $5 \cdot 59$ & $13 \cdot 74$ \\
\hline 19 & 0.75 & $0 \cdot 19$ & $2 \cdot 96$ & 6.98 & $0 \cdot 38$ & $2 \cdot 89$ & $16 \cdot 15$ & 4.94 & $11 \cdot 24$ \\
\hline 1 & 0.71 & 0.58 & 4.45 & $14 \cdot 04$ & $0 \cdot 36$ & 0.58 & 8.67 & 1.65 & 0.64 \\
\hline 1 & $13 \cdot 32^{* * *}$ & 0.42 & $2 \cdot 31$ & $25 \cdot 81^{*}$ & 1.59 & 0.05 & 0.44 & 0.99 & $2 \cdot 58$ \\
\hline 18 & $1 \cdot 26$ & 0.67 & $4 \cdot 34$ & $5 \cdot 30$ & 0.57 & $2 \cdot 58$ & $8 \cdot 74$ & $2 \cdot 25$ & 3.65 \\
\hline 19 & $1 \cdot 80$ & 0.41 & $4 \cdot 11$ & $4 \cdot 60$ & 0.45 & $2 \cdot 44$ & $9 \cdot 87$ & 3.54 & $4 \cdot 48$ \\
\hline
\end{tabular}

(b) the SSD samples

TT1

1. Between reps

2. Between groups (ert vs. nutans)

3. Bet. lines within groups

4. Reps $\times$ lines

TT3

1. Between reps

2. Between groups (ert vs. nutans)

3. Bet. lines within groups

4. Reps $\times$ lines

TT4

1. Between reps

2. Between groups (ert vs. nutans)

3. Bet. lines within groups

4. Reps $\times$ lines

\begin{tabular}{|c|c|c|c|c|c|c|c|c|c|}
\hline 1 & 0.21 & 0.01 & 0.35 & $27 \cdot 68$ & 0.77 & $19 \cdot 42$ & $72 \cdot 24$ & $24 \cdot 56$ & 51.69 \\
\hline 1 & 4.49 & 0.08 & 1.57 & $9 \cdot 20$ & 0.53 & 4.04 & $29 \cdot 31$ & $1 \cdot 06$ & 14.02 \\
\hline 38 & $3 \cdot 35$ & $0 \cdot 20$ & 3.05 & $4 \cdot 74$ & $0 \cdot 37$ & $2 \cdot 89$ & $12 \cdot 24$ & 3.06 & $3 \cdot 71$ \\
\hline 39 & $1 \cdot 26$ & 0.22 & $2 \cdot 26$ & $5 \cdot 68$ & 0.40 & $3 \cdot 11$ & $9 \cdot 97$ & 2.07 & 3.06 \\
\hline 1 & $5 \cdot 25$ & 0.61 & 0.82 & $11 \cdot 62$ & $1 \cdot 73$ & $11 \cdot 77$ & $68 \cdot 74$ & $2 \cdot 74$ & 0.82 \\
\hline 1 & 8.95 & 0.07 & $3 \cdot 72$ & $43 \cdot 55^{*}$ & $0 \cdot 16$ & $9 \cdot 80^{*}$ & $21 \cdot 11$ & $3 \cdot 31$ & $12 \cdot 92$ \\
\hline 38 & 2.97 & 0.27 & $1 \cdot 22$ & $9 \cdot 42$ & 0.52 & 2.09 & $16 \cdot 95$ & $2 \cdot 69$ & $4 \cdot 62$ \\
\hline 39 & 1.09 & $0 \cdot 26$ & $1 \cdot 13$ & $4 \cdot 56$ & $0 \cdot 38$ & $2 \cdot 24$ & $14 \cdot 07$ & $2 \cdot 44$ & $4 \cdot 04$ \\
\hline 1 & 4.02 & 0.76 & 0.19 & $5 \cdot 25$ & 0.01 & $3 \cdot 20$ & $8 \cdot 57$ & $2 \cdot 74$ & 0.31 \\
\hline 1 & $3 \cdot 80$ & 0.13 & 8.66 & 0.82 & 0.30 & $0 \cdot 36$ & $5 \cdot 40$ & 0.35 & 0.84 \\
\hline 38 & $4 \cdot 71$ & $0 \cdot 19$ & $2 \cdot 53$ & $11 \cdot 25$ & 0.63 & $2 \cdot 31$ & $13 \cdot 88$ & $3 \cdot 39$ & $3 \cdot 24$ \\
\hline 39 & $1 \cdot 39$ & 0.11 & 2.97 & $7 \cdot 24$ & 0.40 & 1.91 & $11 \cdot 17$ & $3 \cdot 84$ & $4 \cdot 70$ \\
\hline
\end{tabular}

1980; Powell et al., 1985b). To make joint predictions about mean performance and environmental sensitivity an estimate of the additive genetic correlation is necessary. The between family component of the covariance between mean performance and environmental sensitivity can be used as an approximate estimate of the additive genetic covariance from which we can derive the additive genetic correlation (Powell et al., 1985b). The results for the TT1 and TT3 crosses are given in table 6 . The number of lines that are predicted to fall into four of the possible nine phenotypic classes for combinations of mean performance and environmental sensitivity are tabulated together with the observed number of SSD families. It is clear that the two sets of predictions are in reasonably good agreement with the observed numbers. In fact where there are discrepancies for univariate and bivariate predictions it can invariably be traced back to consistently high estimates of $m$ for environmental sensitivity compared with the midparental value. This may be due to differential survival in the DH samples and or dominance in the case of the $F_{3}$ samples.

\section{CONCLUSIONS}

1. Genotype by environment interactions identified in this study are not necessarily adequately 
Table 5 (a) Estimates of genetical parameters for environmental sensitivity in the TT1 and TT3 crosses

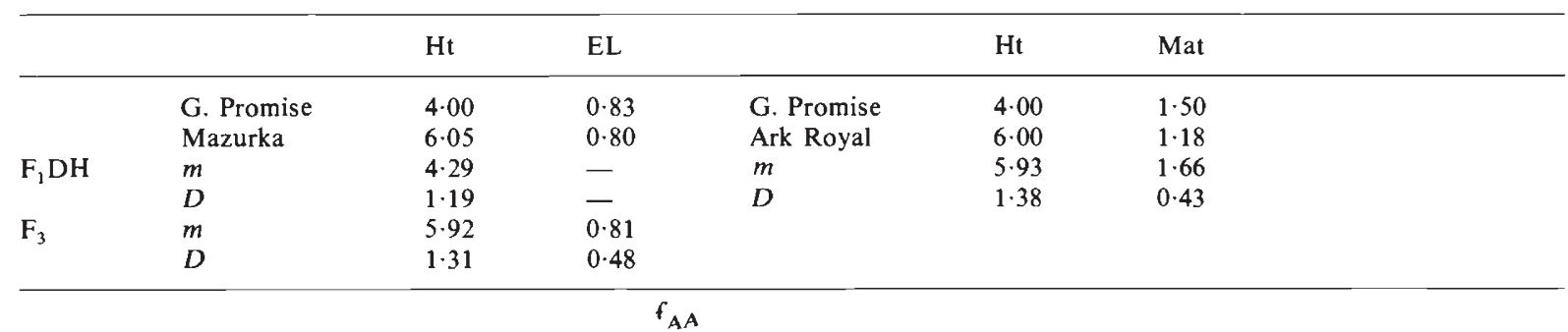

(b) Univariate predictions for environmental sensitivity in the TT1 and TT3 crosses

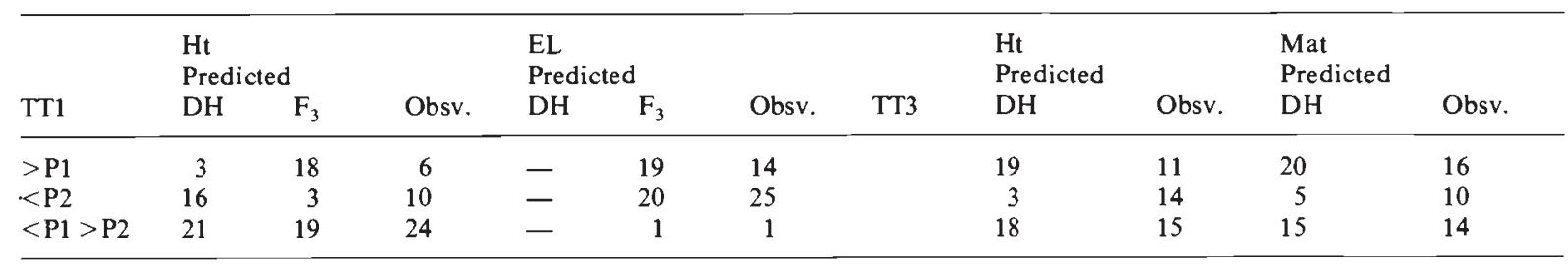

Table 6 Joint predictions for mean performance and environmental sensitivity in the TT1 and TT3 crosses

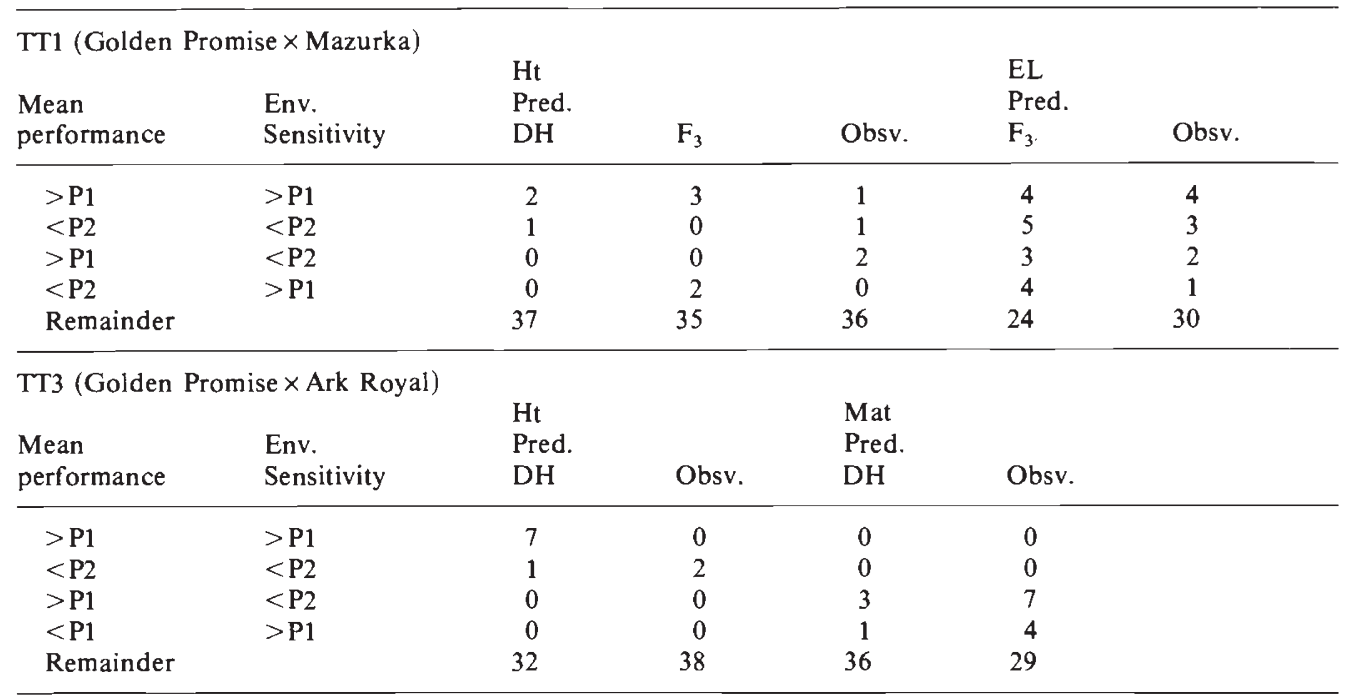

explained by a linear function of the environment. The use of the regression slope to measure environmental sensitivity may be replaced by a genotype's phenotypic variance over environments.

2. Environmental sensitivity measured as a function of a genotype's phenotypic variance is a character which may be included in univariate and bivariate prediction studies. Indeed the present results indicate that $F_{3}$ and $D H$ samples may be used to predict new combinations of mean performance and environmental sensitivity which may appear in SSD populations. Genotypes with higher and lower than average environmental sensitivites may be fixed and selected in recombinant inbred lines.
3. Barley breeding programmes may be organised to produce genotypes with a desired level of sensitivity with the same confidence as is currently applied to mean performance.

\section{REFERENCES}

BREESE, E. L. 1969. The measurement and significance of genotype-environment interactions in grasses. Heredity, 24, 27-44.

CAligari, P. D. S., POWELl, W. AND JINKS, J. L. 1985a. The use of doubled haploids for detecting linkage and pleiotropy between quantitatively varying characters in spring barley. J. Agric. Sci. (in press). 
CAligari, P. D. S., POWELL, W. AND Jinks, J. L. 1985b. The use of doubled haploids in barley breeding. 2. An assessment of univariate cross prediction methods. Heredity, 54, 353-358.

FINLAY, K. W. AND WILKINSON, G. N. 1963. The analysis of adaptation in a plant-breeding programme. Aust. J. Agric. Res., 14, 742-754.

HILL, J. 1975. Genotype-environment interactions-a challenge for plant breeding. J. Agric. Sci. 85, 477-493.

JINKS, J. L. AND CONNOLLY, V. 1975. Determination of the environmental sensitivity of selection lines by the selection environment. Heredity, 34, 401-406.

JINKS, J. L. AND POONI, H. S. 1980. Comparing predictions of mean performance and envirorimental sensitivity of recombinant inbred lines based upon F3 and triple test cross families. Heredity, 45, 305-312.

KALTSIKES, P. J. AND LARTER, E. N. 1970. The interaction of genotype and environment in durum wheat. Euphytica, 19, 236-242.

MATHER, K. 1975. Genotype $x$ environment interactions. II. Some genetical considerations. Heredity, 35, 31-53.
PERKINS, JEAN, M. AND JINKS, J. L. 1968a. Environmental and genotype-environmental components of variability. III Multiple lines and crosses. Heredity, 23, 339-356.

PERKINS, JEAN M., AND JINKS, J. L. 1968b. Environmental and genotype-environmental components of variability. IV. Non-linear interactions for multiple inbred lines. Heredity, $23,525-535$.

POONI, H. S. AND JINKS, J. L. 1978. Predicting the properties of recombinant inbred lines derived by single seed descent for two or more characters simultaneously. Heredity, 40, 349-361.

POWELL, W., THOMAS, W. T. B., CALIGARI, P. D. S. AND JINKS, J. L. 1985a. The effects of major genes on quantitatively varying characters in barley. 1 . The GP ert locus. Heredity, 54, 343-348.

POWELL, W., CALIGARI, P. D. S., MCNICOL, J. W. AND JINKS, J. L. $1985 b$. The use of doubled haploids in barley breeding. 3 . An assessment of multivariate cross prediction methods. Heredity 55, 249-254.

YATES, F. AND COCHRAN, W. G. 1938. The analysis of groups of experiments. J. Agric. Sci. 28, 556-580. 